\title{
On Existence, Uniqueness, and Convergence, of Optimal Control Problems Governed by Parabolic Variational Inequalities
}

\author{
Mahdi Boukrouche ${ }^{1, \star}$ and Domingo A. Tarzia ${ }^{2, \star \star}$ \\ 1 Lyon University UJM F-42023, CNRS UMR 5208, ICJ, France \\ ${ }^{2}$ CONICET and Austral University, Rosario, Argentina
}

\begin{abstract}
I) We consider a system governed by a free boundary problem with Tresca condition on a part of the boundary of a material domain with a source term $g$ through a parabolic variational inequality of the second kind. We prove the existence and uniqueness results to a family of distributed optimal control problems over $g$ for each parameter $h>0$, associated to the Newton law (Robin boundary condition), and of another distributed optimal control problem associated to a Dirichlet boundary condition. We generalize for parabolic variational inequalities of the second kind the Mignot's inequality obtained for elliptic variational inequalities (Mignot, J. Funct. Anal., 22 (1976), 130-185), and we obtain the strictly convexity of a quadratic cost functional through the regularization method for the non-differentiable term in the parabolic variational inequality for each parameter $h$. We also prove, when $h \rightarrow+\infty$, the strong convergence of the optimal controls and states associated to this family of optimal control problems with the Newton law to that of the optimal control problem associated to a Dirichlet boundary condition.

II) Moreover, if we consider a parabolic obstacle problem as a system governed by a parabolic variational inequalities of the first kind then we can also obtain the same results of Part I for the existence, uniqueness and convergence for the corresponding distributed optimal control problems.

III) If we consider, in the problem given in Part I, a flux on a part of the boundary of a material domain as a control variable (Neumann boundary optimal control problem) for a system governed by a parabolic variational inequality of second kind then we can also obtain the existence and uniqueness results for Neumann boundary optimal control problems for each parameter $h>0$, but in this case the convergence when $h \rightarrow+\infty$ is still an open problem.
\end{abstract}

Keywords: Parabolic variational inequalities, convex combination of solutions, regularization method, optimal control problems, strict convexity of cost functional.

\footnotetext{
* Lyon University, UJM F-42023, CNRS UMR 5208, Institut Camille Jordan, 23 Paul Michelon, 42023, Saint-Etienne, France.

** CONICET and Austral University, Mathematics Department, Paraguay 1950, S2000FZF Rosario, Argentina.
}

D. Hömberg and F. Tröltzsch (Eds.): CSMO 2011, IFIP AICT 391, pp. 76-84, 2013.

(C) IFIP International Federation for Information Processing 2013 


\section{Introduction}

The goal of this paper is to show the existence and uniqueness results to a family of distributed (see Sections 2 and 3) or Neumann boundary (see Section 4) optimal control problems for each parameter $h>0$, associated to the Newton law (Robin boundary condition on a part of the boundary of the material domain), and of another distributed optimal control problem associated to a Dirichlet boundary condition. The system of these optimal control problems are governed by free boundary problems (with Tresca boundary condition (see Sections 2 and (4) or of an obstacle type problem (see Section 3) through a parabolic variational inequalities of the first (see Section 3) or second (see Sections 2] and 4) kind 2], 6]. An optimal control problem for elliptic variational inequality of the second kind is given in 9 .

In order to prove the existence and uniqueness results we generalize for parabolic variational inequalities of the second kind the Mignot's inequality obtained for elliptic variational inequalities [18, and then we obtain the strictly convexity of a quadratic cost functional through the regularization method for the non-differentiable term for each parameter $h>0$.

We also prove, when $h \rightarrow+\infty$, the strong convergence of the optimal controls and states associated to this family of optimal control problems with the Newton law to that of the optimal control problem associated to a Dirichlet boundary condition.

We obtain these convergence without using the adjoint state which is a great advantage with respect to the proof given previously for optimal control problems governed by elliptic and parabolic variational equalities [3, 11], 12, [17.

These convergence when $h \rightarrow+\infty$ are valid for the optimal control problems given in Sections 2 and 3, and it is still an open problem for the Neumann boundary optimal control problem given in Section 4

\section{Distributed Optimal Control Problems Governed by Parabolic Variational Inequality of Second Kind}

Let $\Omega$ a bounded open set in $\mathbb{R}^{N}$ with smooth boundary $\partial \Omega=\Gamma_{1} \cup \Gamma_{2}$ such that $\Gamma_{1} \cap \Gamma_{2}=\emptyset$, and meas $\left(\Gamma_{1}\right)>0$. We set $V=H^{1}(\Omega), V_{0}=\left\{v \in V: v_{\Gamma_{1}}=0\right\}$, $H=L^{2}(\Omega), \mathcal{H}=L^{2}(0, T ; H), \mathcal{V}=L^{2}(0, T ; V)$, and the closed convex set $K_{b}=\left\{v \in V: \quad v_{\Gamma_{1}}=b\right\}$. Let given

$$
\begin{array}{r}
b \in L^{2}\left(0, T ; H^{1 / 2}\left(\Gamma_{1}\right)\right), \quad b>0, \quad g \in \mathcal{H}, \quad g \geq 0, \\
q \in L^{2}\left((0, T) \times \Gamma_{2}\right), \quad q>0, \quad u_{b} \in K_{b} .
\end{array}
$$

We consider the following variational problems [6]

Problem 1. Let given $g, q, b$ and $u_{b}$ as in (1). Find $u=u_{g} \in \mathcal{C}(0, T, H) \cap$ $L^{2}\left(0, T ; K_{b}\right)$ with $\dot{u} \in \mathcal{H}$, such that $u(0)=u_{b}$, and solution of the parabolic variational inequality of second kind:

$$
<\dot{u}, v-u>+a(u, v-u)+\Phi(v)-\Phi(u) \geq(g, v-u), \quad \forall v \in K_{b}, \quad t \in(0, T) .
$$


Problem 2. Let given $g, q, b$ and $u_{b}$ as in (1). For all $h>0$, find $u=u_{h g}$ in $\mathcal{C}(0, T, H) \cap \mathcal{V}$ with $\dot{u} \in \mathcal{H}$, such that $u(0)=u_{b}$, and solution of the parabolic variational inequality of second kind

$$
\begin{aligned}
&<\dot{u}, v-u>+ a_{h}(u, v-u)+\Phi(v)-\Phi(u) \geq(g, v-u) \\
&+h \int_{\Gamma_{1}} b(v-u) d s, \forall v \in V, \quad t \in(0, T) .
\end{aligned}
$$

Where $\dot{u}=u_{t},<,>$ denotes the duality brackets between $V^{\prime}$ and $V, a$ is a symmetric, continuous and coercive bilinear form over $V_{0}$, and $\Phi$ is given by

$$
\Phi(v)=\int_{\Gamma_{2}} q|v| d s
$$

and

$$
a(u, v)=\int_{\Omega} \nabla u \nabla v d x, \quad a_{h}(u, v)=a(u, v)+h \int_{\Gamma_{1}} u v d s, \quad(g, v)=\int_{\Omega} g v d x .
$$

Moreover from [15], 20], 21] we have that:

$$
\exists \lambda_{1}>0 \quad \text { such that } \lambda_{h}\|v\|_{V}^{2} \leq a_{h}(v, v) \quad \forall v \in V \text {, with } \lambda_{h}=\lambda_{1} \min \{1, h\}
$$

that is, $a_{h}$ is also a bilinear continuous, symmetric and coercive form on $V$.

We remark that on $\Gamma_{1} \times(0, T)$, Problem 1 is with the Dirichlet condition $u_{\Gamma_{1}}=b$, while Problem 2 is with the Robin's condition $-\nabla u \cdot n=h(u-b)$, where $n$ is the exterior unit vector normal to $\Gamma$. The functional $\Phi$ comes from the Tresca condition on $\Gamma_{2}$ [1, 4].

The existence and uniqueness of the solution to each of the above Problem 1 and Problem 2, is well known see for example [7, 8, [10. Therefore, it allows us to consider $g \mapsto u_{g}$ as a function from $\mathcal{H}$ to $\mathcal{C}(0, T, H) \cap \mathcal{V}$.

Let $M>0$ be a constant and $\mathcal{H}_{+}=\{g \in \mathcal{H}: g \geq 0\}$. We consider the following distributed optimal control problems defined by:

$$
\begin{aligned}
\text { Find } g_{o p} \in \mathcal{H}_{+} \quad \text { such that } \quad J\left(g_{o p}\right) & =\min _{g \in \mathcal{H}_{+}} J(g), \\
\text { Find } g_{o p_{h}} \in \mathcal{H}_{+} \quad \text { such that } \quad J\left(g_{o p_{h}}\right) & =\min _{g \in \mathcal{H}_{+}} J_{h}(g),
\end{aligned}
$$

where the cost functional $J: \mathcal{H} \rightarrow \mathbb{R}$ and $J_{h}: \mathcal{H} \rightarrow \mathbb{R}$ such that [16] (see also [13], 14, 22])

$$
J(g)=\frac{1}{2}\left\|u_{g}\right\|_{\mathcal{H}}^{2}+\frac{M}{2}\|g\|_{\mathcal{H}}^{2}, \quad \text { and } \quad J_{h}(g)=\frac{1}{2}\left\|u_{h g}\right\|_{\mathcal{H}}^{2}+\frac{M}{2}\|g\|_{\mathcal{H}}^{2},
$$

being here $u_{g}, u_{h g}$ the unique solutions of the parabolic variational Problem 1 . and Problem 2 respectively, and corresponding to the control $g$ in $\mathcal{H}$. In order to prove the strict convexity of the cost functional $J$ and $J_{h}$, we generalize for 
parabolic variational inequalities a main property [18 that : For any two control $g_{i} \in \mathcal{H}, i=1$ or $i=2$, we have

$$
\begin{gathered}
u_{\mu g_{1}+(1-\mu) g_{2}} \leq \mu u_{g_{1}}+(1-\mu) u_{g_{2}}, \quad \forall \mu \in[0,1], \\
u_{h\left(\mu g_{1}+(1-\mu) g_{2}\right)} \leq \mu u_{h g_{1}}+(1-\mu) u_{h g_{2}}, \quad \forall \mu \in[0,1],
\end{gathered}
$$

by using a regularization method for the non-differentiable functional $\Phi$ (see [6]). Then we prove the following

Theorem 1. [6] Let $u_{h_{o p_{h}}}, g_{o p_{h}}$ and $u_{g_{o p}}, g_{o p}$ be the states and the optimal controls defined in Problem 1 and Problem 2 respectively. Then, we obtain the following asymptotic behavior:

$$
\begin{gathered}
\lim _{h \rightarrow+\infty}\left\|u_{h g_{o p_{h}}}-u_{g_{o p}}\right\|_{\mathcal{V}}=0, \\
\lim _{h \rightarrow+\infty}\left\|g_{o p_{h}}-g_{o p}\right\|_{\mathcal{H}}=0 .
\end{gathered}
$$

\section{Distributed Optimal Control Problems Governed by Parabolic Variational Inequality of First Kind}

We will examine in this section, some distributed optimal control problems, for which the strong formulation can be linked to a free boundary problems of complementarity type (Obstacle problems [19]), given for example by the following conditions:

$$
\begin{aligned}
& u \geq 0, \quad u(\dot{u}-\Delta u-g)=0, \quad \dot{u}-\Delta u-g \geq 0 \quad \text { in } \Omega, \\
& u=b \geq 0 \text { on } \Gamma_{1}, \quad-\frac{\partial u}{\partial n}=f \text { on } \Gamma_{2}, \quad \text { and } \quad u(0)=u_{b}
\end{aligned}
$$

and

$$
\begin{array}{r}
u \geq 0, \quad u(\dot{u}-\Delta u-g)=0, \quad \dot{u}-\Delta u-g \geq 0 \quad \text { in } \Omega, \\
-\frac{\partial u}{\partial n}=h(u-b) \text { on } \Gamma_{1}, \quad-\frac{\partial u}{\partial n}=f \text { on } \Gamma_{2}, \quad \text { and } \quad u(0)=u_{b}
\end{array}
$$

where $\Omega$ is a multidimentional regular domain whose boundary is $\partial \Omega=\Gamma_{1} \cup \Gamma_{2}$ with $\Gamma_{1} \cap \Gamma_{2}=\emptyset$. Let consider the convex set $K_{b}$ as in Section 2, It is classical that, for a given positive $b \in L^{2}\left(0, T ; H^{\frac{1}{2}}\left(\Gamma_{1}\right)\right), f \in L^{2}\left(0, T ; L^{2}\left(\Gamma_{2}\right)\right)$, and $g \in \mathcal{H}$, the variational formulations of Problems (8)-(9) and (10)-(11) are respectively given by the following parabolic variational problems:

Problem 3. Let given $g, b$ and $u_{b}$ as in (1) and $f \in L^{2}\left(0, T ; L^{2}\left(\Gamma_{2}\right)\right)$. Find $u=u_{g} \in \mathcal{C}(0, T, H) \cap L^{2}\left(0, T ; K_{b}\right)$ with $\dot{u} \in \mathcal{H}$, such that $u(0)=u_{b}$, and

$<\dot{u}, v-u>+a(u, u-v) \geq(g, v-u)-\int_{\Gamma_{2}} f(v-u) d s, \quad \forall v \in K_{b}, \forall t \in(0, T)$. 
Problem 4. Find $u=u_{h g} \in \mathcal{C}(0, T, H) \cap \mathcal{V}$ with $\dot{u} \in \mathcal{H}$, such that $u(0)=u_{b}$, and

$$
\begin{array}{r}
<\dot{u}, v-u>+a_{h}(u, u-v) \geq(g, v-u)+h \int_{\Gamma_{1}} b(v-u) d s \\
-\int_{\Gamma_{2}} f(v-u) d s, \quad \forall v \in V, \quad \forall t \in(0, T) .
\end{array}
$$

where $a$ and $a_{h}$ are as in Section 2, Then the existence and uniqueness of the solution to Problem 3 and Problem 4, is also well known see for example [7], 8], [10]. Then it allows us to consider $g \mapsto u_{g}$ as a function from $\mathcal{H}$ to $\mathcal{C}(0, T, H) \cap \mathcal{V}$. Let $M>0$ be a constant. We consider the same family of distributed optimal control problems (3)-(4) and we obtain the same results of the previous Theorem 1 .

Theorem 2. Let $g, b, u_{b}$ as in (1) and $f \leq 0$ in $\Gamma_{2} \times(0, T)$, we can obtain the same results as in Section [2, for the corresponding distributed optimal control problems (3)-(4) when $g \geq 0$ is the control variable.

\section{Neumann Boundary Optimal Control Problem Governed by Parabolic Variational Inequalities of Second Kind}

We assume in this section that the boundary of a multidimensional regular domain $\Omega$ is decomposed in three parts $\partial \Omega=\Gamma_{1} \cup \Gamma_{2} \cup \Gamma_{3}$ with meas $\left(\Gamma_{1}\right)>0$ and $\operatorname{meas}\left(\Gamma_{3}\right)>0$.

We consider a Neumann boundary optimal control problem whose system is governed by a free boundary problem with Tresca conditions on a portion $\Gamma_{2}$ of the boundary, with the flux $f$ on $\Gamma_{3}$ as the control variable, given by:

Problem 5.

$$
\begin{gathered}
\dot{u}-\Delta u=g \quad \text { in } \quad \Omega \times(0, T), \\
\left|\frac{\partial u}{\partial n}\right|<q \Rightarrow u=0, \text { on } \Gamma_{2} \times(0, T), \\
\left|\frac{\partial u}{\partial n}\right|=q \Rightarrow \exists k>0: \quad u=-k \frac{\partial u}{\partial n}, \text { on } \Gamma_{2} \times(0, T), \\
u=b \quad \text { on } \quad \Gamma_{1} \times(0, T), \\
-\frac{\partial u}{\partial n}=f \quad \text { on } \quad \Gamma_{3} \times(0, T),
\end{gathered}
$$

with the initial condition

$$
u(0)=u_{b} \quad \text { on } \quad \Omega,
$$

and the compatibility condition on $\Gamma_{1} \times(0, T)$

$$
u_{b}=b \quad \text { on } \quad \Gamma_{1} \times(0, T),
$$


where $q>0$ is the Tresca friction coefficient on $\Gamma_{2}$ ([1, [4, [10]). We define the space $\mathcal{F}=L^{2}\left(0, T ; L^{2}\left(\Gamma_{3}\right)\right)$.

The variational formulation of Problem 5 leads to the following parabolic variational problem:

Problem 6. Let given $g, q, b$ and $u_{b}$ as in (11) and $f \in \mathcal{F}, f \leq 0$. Find $u=u_{f}$ in $\mathcal{C}(0, T, H) \cap L^{2}\left(0, T ; K_{b}\right)$ with $\dot{u} \in \mathcal{H}$, such that $u(0)=u_{b}$, and for $t \in(0, T)$

$<\dot{u}, v-u>+a(u, u-v)+\Phi(v)-\Phi(u) \geq(g, v-u)-\int_{\Gamma_{3}} f(v-u) d s, \forall v \in K_{b}$.

where $a$ and $\Phi$ are defined as in Section 2

We consider also the following problem where we change, in Problem 5, only the Dirichlet condition on $\Gamma_{1} \times(0, T)$ by the Newton law or a Robin boundary condition.

Problem 7.

$$
\begin{gathered}
\dot{u}-\Delta u=g \quad \text { in } \quad \Omega \times(0, T), \\
\left|\frac{\partial u}{\partial n}\right|<q \Rightarrow u=0, \text { on } \Gamma_{2} \times(0, T), \\
\left|\frac{\partial u}{\partial n}\right|=q \Rightarrow \exists k>0: \quad u=-k \frac{\partial u}{\partial n}, \text { on } \Gamma_{2} \times(0, T), \\
-\frac{\partial u}{\partial n}=h(u-b) \quad \text { on } \quad \Gamma_{1} \times(0, T), \\
-\frac{\partial u}{\partial n}=f \quad \text { on } \quad \Gamma_{3} \times(0, T),
\end{gathered}
$$

with the initial condition

$$
u(0)=u_{b} \quad \text { on } \quad \Omega,
$$

and the condition of compatibility on $\Gamma_{1} \times(0, T)$

$$
u_{b}=b \quad \text { on } \quad \Gamma_{1} \times(0, T) .
$$

The variational formulation of the problem (77) leads to the the following parabolic variational problem

Problem 8. Let given $g, q, b, u_{b}$ and $f$ as in Problem 6 . For all $h>0$, find $u=u_{h f} \in \mathcal{C}(0, T, H) \cap \mathcal{V}$ with $\dot{u} \in \mathcal{H}$, such that $u(0)=u_{b}$, and for $t \in(0, T)$

$$
\begin{aligned}
<\dot{u}, v-u>+a_{h}(u, u-v)+\Phi(v)-\Phi(u) & \geq(g, v-u)-\int_{\Gamma_{3}} f(v-u) d s \\
& +h \int_{\Gamma_{1}} b(v-u) d s, \quad \forall v \in V,
\end{aligned}
$$

where $a_{h}$ and $\Phi$ are defined as in Section 2 


\subsection{Neumann Boundary Optimal Control Problems}

Let $M>0$ be a constant and we define the space $\mathcal{F}_{-}=\{f \in \mathcal{F}: f \leq 0\}$. We consider the new following Neumannn boundary optimal control problems defined by:

Problem 9. Find the optimal control $f_{\text {op }} \in \mathcal{F}_{-}$such that

$$
J\left(f_{o p}\right)=\min _{f \in \mathcal{F}_{-}} J(f)
$$

where the cost functional $J: \mathcal{F} \rightarrow \mathbb{R}_{0}^{+}$is given by

$$
J(f)=\frac{1}{2}\left\|u_{f}\right\|_{\mathcal{H}}^{2}+\frac{M}{2}\|f\|_{\mathcal{F}}^{2} \quad(M>0)
$$

and $u_{f}$ is the unique solution of the Problem 6 .

Problem 10. Find the optimal control $f_{o p_{h}} \in \mathcal{F}_{-}$such that

$$
J\left(f_{o p_{h}}\right)=\min _{f \in \mathcal{F}_{-}} J_{h}(f)
$$

where the cost functional $J_{h}: \mathcal{F} \rightarrow \mathbb{R}_{0}^{+}$is given by

$$
J_{h}(f)=\frac{1}{2}\left\|u_{h f}\right\|_{\mathcal{H}}^{2}+\frac{M}{2}\|f\|_{\mathcal{F}}^{2} \quad(M>0, \quad h>0)
$$

and $u_{h f}$ is the unique solution of Problem 8 .

Theorem 3. Under the assumptions given in Problem [6, we have the following properties:

a) The cost functional $J$ is strictly convex on $\mathcal{F}_{-}$,

b) There exists a unique optimal $f_{\text {op }} \in \mathcal{F}_{-}$solution of the new Neumannn boundary optimal control Problem 9.

Proof. We give some sketch of the proof.

i) We generalize for parabolic variational inequalities of the second kind the estimates obtained for convex combination of solutions for elliptic variational inequalities [5] that is, the estimate between

$$
u_{4}(\mu)=u_{\mu f_{1}+(1-\mu) f_{2}}, \text { and } u_{3}(\mu)=\mu u_{f_{1}}+(1-\mu) u_{f_{2}},
$$

for any two element $f_{1}$ and $f_{2}$ in $\mathcal{F}$.

ii) The main difficulty, to prove this result comes from the fact that the functional $\Phi$ is not differentiable. To overcome this difficulty, we use the regularization method and consider for $\varepsilon>0$ the following approach of $\Phi$ defined by:

$$
\Phi_{\varepsilon}(v)=\int_{\Gamma_{2}} q \sqrt{\varepsilon^{2}+|v|^{2}} d s, \quad \forall v \in V,
$$


which is Gateaux differentiable, with

$$
\left\langle\Phi_{\varepsilon}^{\prime}(w), v\right\rangle=\int_{\Gamma_{2}} \frac{q w v}{\sqrt{\varepsilon^{2}+|w|^{2}}} d s \quad \forall(w, v) \in V^{2} .
$$

We define $u^{\varepsilon}$ as the unique solution of the corresponding parabolic variational inequality for all $\varepsilon>0$. We obtain that for all $\mu \in[0,1]$ we have $u_{4}^{\varepsilon}(\mu) \leq u_{3}^{\varepsilon}(\mu)$ for all $\varepsilon>0$.

iii) When $\varepsilon \rightarrow 0$ we have that:

$$
u_{i}^{\varepsilon} \rightarrow u_{i} \text { strongly in } \mathcal{V} \cap L^{\infty}(0, T ; H) \text { for } i=1,2,3,4,
$$

for all $\mu \in[0,1]$ and therefore we get:

$$
0 \leq u_{4}(\mu) \leq u_{3}(\mu) \quad \text { in } \quad \Omega \times[0, T], \quad \forall \mu \in[0,1]
$$

iv) For all $\mu \in] 0,1\left[\right.$, and for all $f_{1}, f_{2}$ in $\mathcal{F}$, and by using $f_{3}(\mu)=\mu f_{1}+(1-\mu) f_{2}$ we obtain that:

$$
\begin{aligned}
\mu J\left(f_{1}\right)+(1-\mu) J\left(f_{2}\right)-J\left(f_{3}(\mu)\right)=\frac{1}{2}\left(\left\|u_{3}(\mu)\right\|_{\mathcal{H}}^{2}-\left\|u_{4}(\mu)\right\|_{\mathcal{H}}^{2}\right) \\
+\frac{1}{2} \mu(1-\mu)\left\|u_{f_{1}}-u_{f_{2}}\right\|_{\mathcal{H}}^{2}+\frac{M}{2} \mu(1-\mu)\left\|f_{1}-f_{2}\right\|_{\mathcal{F}}^{2} .
\end{aligned}
$$

Then $J$ is strictly convex functional on $\mathcal{F}_{-}$and therefore there exists a unique optimal $f_{o p} \in \mathcal{F}_{-}$solution of the new Neumannn boundary optimal control Problem 9.

Theorem 4. Under the assumptions given in Problem 6 , we have the following properties:

a) The cost functional $J_{h}$ are strictly convex on $\mathcal{F}_{-}$, for all $h>0$,

b) There exists a unique optimal $f_{o p_{h}} \in \mathcal{F}_{-}$solution of the new Neumannn boundary optimal control Problem 10, for all $h>0$.

Proof. We follow a similar method to the one developed in Theorem 3 for all $h>0$.

\subsection{Open Problem}

The convergence of the new Neumann boundary optimal control Problem 10 to the new Neumann boundary optimal control Problem 9 when $h \rightarrow \infty$ is an open problem.

Acknowledgements. The autors would like to thank very much the unknown referee for helpful comments which allowed to improve the paper. This paper was partially sponsored by the Institut Camille Jordan ST-Etienne University for first author and the project PICTO Austral \# 73 from ANPCyT and Grant AFOSR FA9550-10-1-0023 for the second author. 


\section{References}

1. Amassad, A., Chenais, D., Fabre, C.: Optimal control of an elastic contact problem involving Tresca friction law. Nonlinear Analysis 48, 1107-1135 (2002)

2. Barbu, V.: Optimal control of variational inequalities. Research Notes in Mathematics, vol. 100. Pitman (Advanced Publishing Program), Boston (1984)

3. Ben Belgacem, F., El Fekih, H., Metoui, H.: Singular perturbation for the Dirichlet boundary control of elliptic problems. ESAIM: M2AN 37, 833-850 (2003)

4. Boukrouche, M., El Mir, R.: On a non-isothermal, non-Newtonian lubrication problem with Tresca law: Existence and the behavior of weak solutions. Nonlinear Analysis: Real World Applications 9(2), 674-692 (2008)

5. Boukrouche, M., Tarzia, D.A.: On a convex combination of solutions to elliptic variational inequalities. Electro. J. Diff. Equations (31), 1-10 (2007)

6. Boukrouche, M., Tarzia, D.A.: Convergence of distributed optimal controls for second kind parabolic variational inequalities. Nonlinear Analysis: Real World Applications 12(4), 2211-2224 (2011)

7. Brézis, H.: Problèmes unilatéraux. J. Math. Pures Appl. 51, 1-162 (1972)

8. Chipot, M.: Elements of nonlinear Analysis. Birkhäuser Advanced Texts (2000)

9. De Los Reyes, J.C.: Optimal control of a class of variational inequalities of the second kind. SIAM J. Control Optim. 49, 1629-1658 (2011)

10. Duvaut, G., Lions, J.L.: Les inéquations en Mécanique et en Physique. Dunod, Paris (1972)

11. Gariboldi, C.M., Tarzia, D.A.: Convergence of distributed optimal controls on the internal energy in mixed elliptic problems when the heat transfer coefficient goes to infinity. Appl. Math. Optim. 47(3), 213-230 (2003)

12. Gariboldi, C.M., Tarzia, D.A.: Convergence of boundary optimal controls problems with restrictions in mixed elliptic Stefan-like problems. Adv. Diff. Eq. and Control Processes 1, 113-132 (2008)

13. Kesavan, S., Muthukumar, T.: Low-cost control problems on perforated and nonperforated domains. Proc. Indian Acad. Sci. (Math. Sci.) 118(1), 133-157 (2008)

14. Kesavan, S., Saint Jean Paulin, J.: Optimal control on perforated domains. J. Math. Anal. Appl. 229, 563-586 (1997)

15. Kinderlehrer, D., Stampacchia, G.: An introduction to variational inequalities and their applications. Academic Press, New York (1980)

16. Lions, J.L.: Contrôle optimal de systèmes gouvernés par des équations aux dérivées partielles. Dunod, Paris (1968)

17. Menaldi, J.L., Tarzia, D.A.: A distributed parabolic control with mixed boundary conditions. Asymptotic Anal. 52, 227-241 (2007)

18. Mignot, F.: Contrôle dans les inéquations variationelles elliptiques. J. Functional Anal. 22(2), 130-185 (1976)

19. Rodrigues, J.F.: Obstacle problems in mathematical physics. North-Holland, Amsterdam (1987)

20. Tabacman, E.D., Tarzia, D.A.: Sufficient and/or necessary condition for the heat transfer coefficient on $\Gamma_{1}$ and the heat flux on $\Gamma_{2}$ to obtain a steady-state two-phase Stefan problem. J. Diff. Equations 77(1), 16-37 (1989)

21. Tarzia, D.A.: Una familia de problemas que converge hacia el caso estacionario del problema de Stefan a dos fases. Math. Notae 27, 157-165 (1979)

22. Tröltzsch, F.: Optimal control of partial differential equations: Theory, methods and applications. American Math. Soc., Providence (2010) 